\title{
GENERALIZED CONVEX FUNCTIONS
}

BY E. F. BECKENBACH

1. Introduction. We shall be concerned in this paper with real finite functions $f(x)$ defined in an interval $a<x<b$. A function $f(x)$ is said to be convex* in $(a, b)$ provided, for an arbitrary subinterval $\left(x_{1}, x_{2}\right)$ interior to $(a, b)$, the curve $y=f(x)$ lies nowhere above the line segment joining its end points; that is, provided, for arbitrary $x_{1}, x_{2}, x$, with $a<x_{1}<x<x_{2}<b$,

$$
f(x) \leqq F_{12}(x),
$$

where $F_{12}(x)$ is the function of the form

$$
F_{12}(x)=\alpha x+\beta
$$

satisfying

$$
F_{12}\left(x_{1}\right)=f\left(x_{1}\right), \quad F_{12}\left(x_{2}\right)=f\left(x_{2}\right) .
$$

Several generalizations of the notion of a convex function to other classes of functions of one variable have found their way into the literature. $\dagger$ In what follows we discuss the notions which seem to underlie these classes of functions.

Let $F(x ; \alpha, \beta)$ be a (two-parameter) family of real finite functions defined for $a<x<b$ and satisfying the following conditions:

(1) each $F(x ; \alpha, \beta)$ is a continuous function of $x$;

(2) there is a unique member of the family which, at arbitrary $x_{1}, x_{2}$ satisfying $a<x_{1}<x_{2}<b$, takes on arbitrary values $y_{1}, y_{2}$.

For example, such a simple $F(x ; \alpha, \beta)$ as $x^{2}+\alpha x+\beta$ is not in-

* J. L. W. V. Jensen, Sur les fonctions convexes et les inégalités entre les valeurs moyennes, Acta Mathematica, vol. 30 (1906), pp. 175-193.

$\dagger$ See E. Phragmén and E. Lindelöf, Sur une extension d'un principe classique de l'analyse, Acta Mathematica, vol. 31 (1908), pp. 381-406; G. P6́lya, Untersuchungen ilber Lücken und Singularitäten von Potenzreihen, Mathematische Zeitschrift, vol. 29 (1929), pp. 549-640; B. Jessen, Über die Verallgemeinerungen des arithmetischen Mittels, Acta Szeged, vol. 5 (1931), pp. 108116; G. Valiron, Fonctions convexes et fonctions entières, Bulletin de la Société Mathématique de France, vol. 60 (1932), pp. 278-287. 
cluded in any of the above mentioned generalizations. Other examples are $\alpha x+\beta ; A x^{3}+B x^{2}+\alpha x+\beta$, where $A$ and $B$ are fixed constants; $\alpha e^{\rho x}+\beta e^{-\rho x}$, where $\rho$ is a fixed constant; and, with $b-a \leqq \pi / \rho, \alpha \sin \rho x+\beta \cos \rho x$. Still another example of the family $F(x ; \alpha, \beta)$ is the set of images of all non-vertical straight lines, under a one-to-one continuous transformation of the domain $a<x<b$ of the plane into itself in such a way that every vertical line is transformed into itself.

Members of the above family shall be denoted simply by $F(x)$, not $F(x ; \alpha, \beta)$, individual members being distinguished by subscripts. In particular, $F_{i j}(x)$ shall denote the member satisfying

$$
F_{i j}\left(x_{i}\right)=f\left(x_{i}\right), \quad F_{i j}\left(x_{j}\right)=f\left(x_{j}\right), \quad\left(a<x_{i}<x_{j}<b\right) .
$$

Definition 1. The function $f(x)$ shall be called a $s u b-F(x ; \alpha, \beta)$ function, or simply a $s u b-F(x)$ function, provided

$$
f(x) \leqq F_{12}(x)
$$

for all $x_{1}, x_{2}, x$, with $a<x_{1}<x<x_{2}<b$.

Definition 2. Super-F(x) functions are defined exactly as are sub- $F(x)$ functions, excepting that the sign of inequality is reversed; the analysis is the same, mutatis mutandis.

2. The Family $F(x ; \alpha, \beta)$. We shall prove the following theorem.

Theorem 1. Let $a<x_{0}<b$, and let $F_{r}(x), F_{s}(x)$ be two members of the family satisfying (1) and (2) such that

$$
\begin{aligned}
F_{r}\left(x_{0}\right) & =F_{s}\left(x_{0}\right), \\
F_{r}(x) & \not \equiv F_{s}(x), \quad(a<x<b) ;
\end{aligned}
$$

then $F_{r}(x)>F_{s}(x)$ for all $x$ in $(a, b)$ on one side of $x_{0}$, while $F_{r}(x)<F_{s}(x)$ for all $x$ in $(a, b)$ on the other side of $x_{0}$.

Proof. By (2), (4), and (5), $F_{r}(x) \neq F_{s}(x),(a<x<b)$, except at $x_{0}$; consequently, by $(1)$, on either side of $x_{0}$, one of $F_{r}(x)$, $F_{s}(x)$ is greater than the other. Suppose it could be the same one, say $F_{s}(x)$, which is greater on each side; we shall show this is impossible.

Let $a<x_{1}<x_{0}<x_{2}<b$, and consider $F_{t}(x)$, determined by 


$$
F_{t}\left(x_{1}\right)=F_{s}\left(x_{1}\right), \quad F_{t}\left(x_{2}\right)=F_{r}\left(x_{2}\right) .
$$

Then $F_{t}\left(x_{2}\right)<F_{s}\left(x_{2}\right)$, so that $F_{t}(x)<F_{s}(x),\left(x_{1}<x<b\right)$; in particular,

$$
F_{t}\left(x_{0}\right)<F_{s}\left(x_{0}\right)
$$

Similarly,

$$
F_{t}\left(x_{0}\right)>F_{r}\left(x_{0}\right) \text {. }
$$

Now (6) and (7) contradict (4), establishing the theorem.

Corollary 1. If $a<x_{1}<x_{2}<b$, and if $F_{r}\left(x_{1}\right)<F_{s}\left(x_{1}\right), F_{r}\left(x_{2}\right)$ $<F_{s}\left(x_{2}\right)$, then $F_{r}(x)<F_{s}(x)$ for $x_{1} \leqq x \leqq x_{2} ;$ but if $F_{r}\left(x_{1}\right)<F_{s}\left(x_{1}\right)$, $F_{r}\left(x_{2}\right)>F_{s}\left(x_{2}\right)$, then $F_{r}(x)<F_{s}(x)$ for $a<x \leqq x_{1}$, and $F_{r}(x)>F_{s}(x)$ for $x_{2} \leqq x<b$.

THEOREM 2. Let the points $\left(x_{n}, y_{n}\right),\left(x_{n}{ }^{\prime}, y_{n}{ }^{\prime}\right),(n=0,1,2, \cdots)$, satisfy $a<x_{n}<x_{n}^{\prime}<b$, and

$$
\lim _{n \rightarrow \infty}\left(x_{n}, y_{n}\right)=\left(x_{0}, y_{0}\right), \quad \lim _{n \rightarrow \infty}\left(x_{n}^{\prime}, y_{n}^{\prime}\right)=\left(x_{0}^{\prime}, y_{0}^{\prime}\right),
$$

and let $F_{n}(x)$ be determined by

$$
F_{n}\left(x_{n}\right)=y_{n}, \quad F_{n}\left(x_{n}^{\prime}\right)=y_{n}^{\prime} ;
$$

then

$$
\lim _{n \rightarrow \infty} F_{n}(x)=F_{0}(x), \quad(a<x<b),
$$

uniformly in any closed subinterval of $a<x<b$.

Proof. For a given $\epsilon>0$, and for an arbitrary $\bar{x}$ in $(a, b)$, with $\bar{x} \neq x_{0}, \bar{x} \neq x_{0}^{\prime}$, let the functions $F_{p}(x), \cdots, F_{s}(x)$ be determined by

(10) $F_{p}\left(x_{0}\right)=F_{0}\left(x_{0}\right)$,

(11) $F_{p}(\bar{x})=F_{0}(\bar{x})+\epsilon$,

(12) $F_{q}\left(x_{0}\right)=F_{0}\left(x_{0}\right)$,

(13) $F_{q}(\bar{x})=F_{0}(\bar{x})-\epsilon$,

(14) $F_{r}\left(x_{0}^{\prime}\right)=F_{p}\left(x_{0}^{\prime}\right)$,

(15) $F_{r}(\bar{x})=F_{p}(\bar{x})+\epsilon=F_{0}(\bar{x})+2 \epsilon$,

(16) $F_{s}\left(x_{0}^{\prime}\right)=F_{q}\left(x_{0}^{\prime}\right)$,

(17) $F_{s}(\bar{x})=F_{q}(\bar{x})-\epsilon=F_{0}(\bar{x})-2 \epsilon$.

Let $\delta$ denote the least of the four (minimum) distances from the curves $y=F_{r}(x)$ and $y=F_{s}(x)$ to the points $\left(x_{0}, y_{0}\right)$ and $\left(x_{0}^{\prime}, y_{0}^{\prime}\right)$. It is easily verified, by Theorem 1 and (10)-(17), that $\delta>0$. Let 


$$
\delta^{*}=\min \left(\delta,\left|\bar{x}-\bar{x}_{0}\right|,\left|\bar{x}-x_{0}^{\prime}\right|\right) \text {; }
$$

then $\delta^{*}>0$.

Now let $n_{0}=n_{0}(\bar{x})$ be so large that

$$
\begin{aligned}
&\left\{\left(x_{n}-x_{0}\right)^{2}+\left(y_{n}-y_{0}\right)^{2}\right\}^{1 / 2}<\delta^{*}, \\
&\left\{\left(x_{n}^{\prime}-x_{0}^{\prime}\right)^{2}+\left(y_{n}^{\prime}-y_{0}^{\prime}\right)^{2}\right\}^{1 / 2}<\delta^{*}, \quad\left(n \geqq n_{0}\right) .
\end{aligned}
$$

We shall prove that

$$
\left|F_{n}(\bar{x})-F_{0}(\bar{x})\right|<2 \epsilon, \quad\left(n \geqq n_{0}\right) .
$$

Three cases arise, as follows.

CAsE 1. $x_{0}<\bar{x}<x_{0}^{\prime}$. In this case, by (10), (11), and Theorem 1 , $F_{p}\left(x_{0}^{\prime}\right)>F_{0}\left(x_{0}^{\prime}\right)$; consequently, by (10), (14), (15), and Theorem $1, F_{r}\left(x_{0}\right)>F_{0}\left(x_{0}\right), F_{r}\left(x_{0}^{\prime}\right)>F_{0}\left(x_{0}^{\prime}\right)$. Similarly, $F_{s}\left(x_{0}\right)<F_{0}\left(x_{0}\right)$, $F_{s}\left(x_{0}^{\prime}\right)<F_{0}\left(x_{0}^{\prime}\right)$. By (1) and (19), then, for $n \geqq n_{0}$,

$$
\begin{gathered}
F_{s}\left(x_{n}\right)<y_{n}=F_{n}\left(x_{n}\right)<F_{r}\left(x_{n}\right), \\
F_{s}\left(x_{n}^{\prime}\right)<y_{n}^{\prime}=F_{n}\left(x_{n}^{\prime}\right)<F_{r}\left(x_{n}^{\prime}\right),
\end{gathered}
$$

whence it follows from Corollary 1 that

$$
F_{s}(x)<F_{n}(x)<F_{r}(x), \quad\left(x_{n}<x<x_{n}^{\prime}, n \geqq n_{0}\right) .
$$

In particular,

$$
F_{s}(\bar{x})<F_{n}(\bar{x})<F_{r}(\bar{x}), \quad\left(n \geqq n_{0}\right) .
$$

Now (20) follows from (15), (17) and (22).

CAse 2. $x_{0}^{\prime}<\bar{x}<b$. The above analysis gives this time

$$
F_{r}\left(x_{0}\right)<F_{0}\left(x_{0}\right)<F_{s}\left(x_{0}\right), \quad F_{r}\left(x_{0}^{\prime}\right)>F_{0}\left(x_{0}^{\prime}\right)>F_{s}\left(x_{0}^{\prime}\right),
$$

and therefore, for $n \geqq n_{0}$,

$$
\begin{gathered}
F_{s}\left(x_{n}\right)>y_{n}=F_{n}\left(x_{n}\right)>F_{r}\left(x_{n}\right), \\
F_{s}\left(x_{n}^{\prime}\right)<y_{n}^{\prime}=F_{n}\left(x_{n}^{\prime}\right)<F_{r}\left(x_{n}^{\prime}\right),
\end{gathered}
$$

whence it follows from Corollary 1 that

$(21, \mathrm{~B}) \quad F_{s}(x)<F_{n}(x)<F_{r}(x), \quad x_{n}^{\prime}<x<b, \quad\left(n \geqq n_{0}\right)$.

In particular, (22), and therefore (20), hold in this case.

CAsE 3. $a<\bar{x}<x_{0}$. The same analysis gives now 


$$
F_{r}\left(x_{0}\right)>F_{0}\left(x_{0}\right)>F_{s}\left(x_{0}\right), \quad F_{r}\left(x_{0}^{\prime}\right)<F_{0}\left(x_{0}^{\prime}\right)<F_{s}\left(x_{0}^{\prime}\right),
$$

and therefore, for $n \geqq n_{0}$,

$$
\begin{gathered}
F_{s}\left(x_{n}\right)<y_{n}=F_{n}\left(x_{n}\right)<F_{r}\left(x_{n}\right), \\
F_{s}\left(x_{n}^{\prime}\right)>y_{n}^{\prime}=F_{n}\left(x_{n}^{\prime}\right)>F_{r}\left(x_{n}^{\prime}\right),
\end{gathered}
$$

whence

$$
F_{s}(x)<F_{n}(x)<F_{r}(x), \quad\left(a<x<x_{n} ; n \geqq n_{0}\right),
$$

so that again (22) and (20) hold.

For each $x$ in $(a, b)$, except for $x_{0}, x_{0}^{\prime},(9)$ follows from (20). Let $x_{t}, x_{t}^{\prime}$, with $x_{t}<x_{t}^{\prime}$, be any other two points in $(a, b)$. Then the sequences

$$
\left(x_{t}, F_{n}\left(x_{t}\right)\right), \quad\left(x_{t}^{\prime}, F_{n}\left(x_{t}^{\prime}\right)\right), \quad(n=1,2,3, \cdots),
$$

satisfy, by the above analysis,

$$
\begin{gathered}
\lim _{n \rightarrow \infty}\left(x_{t}, F_{n}\left(x_{t}\right)\right)=\left(x_{t}, F_{0}\left(x_{t}\right)\right), \\
\lim _{n \rightarrow \infty}\left(x_{t}^{\prime}, F_{n}\left(x_{t}^{\prime}\right)\right)=\left(x_{t}^{\prime}, F_{0}\left(x_{t}^{\prime}\right)\right) .
\end{gathered}
$$

Now since (24) holds, the above analysis can be applied to the sequences (23), giving (20) this time for $\bar{x} \neq x_{t}, \bar{x}=x_{t}^{\prime}$, in particular for $\bar{x}=x_{0}$ and for $\bar{x}=x_{0}^{\prime}$. Therefore (20) and (9) hold throughout $(a, b)$.

In each of the above three cases, by (1), there is an interval $\bar{x}-\eta<x<\bar{x}+\eta$, where $\eta=\eta(\bar{x})>0$, in which

$F_{s}(x)>F_{s}(\bar{x})-\epsilon, \quad F_{r}(x)<F_{r}(\bar{x})+\epsilon, \quad\left|F_{0}(x)-F_{0}(\bar{x})\right|<\epsilon$, and therefore, by (15), (17), and (21, A, B, C), in which

$$
\begin{aligned}
& \left|F_{n}(x)-F_{0}(x)\right|<4 \epsilon, \\
& \quad\left(\bar{x}-\eta(\bar{x})<x<\bar{x}+\eta(\bar{x}) ; n \geqq n_{0}(\bar{x})\right) .
\end{aligned}
$$

The same discussion holds, according to the preceding paragraph, for intervals about $x_{0}, x_{0}^{\prime}$. Then (25) holds for arbitrary $\bar{x}$, with $a<\bar{x}<b$, whence the uniformity of (9) for any closed subinterval of $a<x<b$ follows from a simple application of the familiar Heine-Borel Theorem.

Corollary 2. Any subset of the family $F(x ; \alpha, \beta)$ is compact, provided the ordinates are bounded for two distinct abscissas. 
Proof. One readily sets up sequences satisfying the conditions of Theorem 2.

3. Some Properties of Sub-F(x; $\alpha, \beta)$ Functions.

THEOREM 3. If $f(x)$ is a sub-F(x) function, then for any $x_{1}, x_{2}$, with $a<x_{1}<x_{2}<b$, the inequality $f(x) \geqq F_{12}(x)$ holds for $a<x<x_{1}$, and for $x_{2}<x<b$.

Proof. Fix $x_{3}$, with $x_{2}<x_{3}<b$. Since

$$
F_{13}\left(x_{1}\right)=F_{12}\left(x_{1}\right)=f\left(x_{1}\right), \quad F_{13}\left(x_{2}\right) \geqq F_{12}\left(x_{2}\right)=f\left(x_{2}\right),
$$

it follows from Theorem 1 that

$$
f\left(x_{3}\right)=F_{13}\left(x_{3}\right) \geqq F_{12}\left(x_{3}\right) .
$$

A similar proof holds for $a<x_{3}<x_{1}$.

Theorem 4. If $f(x)$ is a sub-F(x) function, and if, for some $x_{1}, x_{2}, x_{3}$, with $a<x_{1}<x_{3}<x_{2}<b$,

$$
f\left(x_{3}\right)=F_{12}\left(x_{3}\right)
$$

then $f(x) \equiv F_{12}(x),\left(x_{1} \leqq x \leqq x_{2}\right)$.

Proof. By (2) and (26),

$$
F_{12}(x) \equiv F_{13}(x) \equiv F_{32}(x) ;
$$

consequently, by Theorem 3 and (27),

$$
\begin{aligned}
& F_{12}(x) \equiv F_{13}(x) \leqq f(x), \quad\left(x_{3} \leqq x<b\right), \\
& F_{12}(x) \equiv F_{32}(x) \leqq f(x), \quad\left(a<x \leqq x_{3}\right) .
\end{aligned}
$$

But according to (3),

$$
F_{12}(x) \geqq f(x), \quad\left(x_{1} \leqq x \leqq x_{2}\right) .
$$

The theorem follows from inequalities (28), (29), and (30).

THEOREM 5. If $f(x)$ is a sub- $F(x)$ function, and if $a<x_{3} \leqq x_{1}<x_{2}$ $\leqq x_{4}<b$, then

$$
F_{34}(x) \geqq F_{12}(x), \quad\left(x_{3} \leqq x \leqq x_{4}\right) .
$$

Proof. By Theorem 3,

$$
F_{34}\left(x_{3}\right)=f\left(x_{3}\right) \geqq F_{12}\left(x_{3}\right), \quad F_{34}\left(x_{4}\right)=f\left(x_{4}\right) \geqq F_{12}\left(x_{4}\right),
$$


whence, by Theorem 1 and its corollary, (31) follows. Incidentally, by (2) and Theorem 1, the sign of equality in (31) holds nowhere in $x_{3} \leqq x \leqq x_{4}$, except at most at one of the end points, unless it holds identically.

THEOREM 6. If $f(x)$ is a sub-F(x) function, then $f(x)$ is continuous.

Proof. We shall show that $f(x)$ is continuous at an arbitrary $x_{0}$, with $a<x_{0}<b$. Let $a<x_{1}<x_{0}<x_{2}<b$. Then, by Definition 1 and Theorem 3 , for $x_{1}<x_{0}-h<x_{0}+h<x_{2}$,

$$
\begin{aligned}
& F_{10}\left(x_{0}-h\right) \geqq f\left(x_{0}-h\right) \geqq F_{02}\left(x_{0}-h\right), \\
& F_{10}\left(x_{0}+h\right) \leqq f\left(x_{0}+h\right) \leqq F_{02}\left(x_{0}+h\right) .
\end{aligned}
$$

Let $h \rightarrow 0$; the theorem follows from (1).

REMARK. Though a convex function necessarily possesses a derivative almost everywhere, not all sub- $F(x)$ functions possess this property.

Proof. Let $\phi(x)$ be a nowhere differentiable function; the family

$$
F(x ; \alpha, \beta) \equiv \phi(x)+\alpha x+\beta
$$

satisfies conditions (1) and (2). Now any particular member of this family is itself a sub- $F(x)$ function, but is nowhere differentiable.

4. Characterization of $\operatorname{Sub}-F(x ; \alpha, \beta)$ Functions.

THEOREM 7. A necessary and sufficient condition that a continuous function $f(x)$ be a sub- $F(x)$ function is that for all $x_{0}$, with $a<x_{0}<b$, and for all $\delta>0$, there exist a positive $h=h\left(x_{0}, \delta\right)<\delta$, with

$$
a<x_{1}=x_{0}-h<x_{0}<x_{0}+h=x_{2}<b,
$$

such that

$$
f\left(x_{0}\right) \leqq F_{12}\left(x_{0}\right) .
$$

NeCEssity. If $f(x)$ is a sub- $F(x)$ function, then for all $h$ such that (32) is satisfied, (33) holds as a special case of (3). 
Sufficiency. If $f(x)$ is not a sub- $F(x)$ function, then by (3) there exist $x_{3}, x_{4}, x_{5}$, with $a<x_{3}<x_{5}<x_{4}<b$, such that

$$
f\left(x_{5}\right)>F_{34}\left(x_{5}\right) \text {. }
$$

By (34) and the continuity of $f(x)$ and of $F_{34}(x)$, there exist $x_{1}, x_{2}$, with $x_{3} \leqq x_{1}<x_{5}<x_{2} \leqq x_{4}$, such that

$$
f(x) \geqq F_{34}(x), \quad\left(x_{1} \leqq x \leqq x_{2}\right),
$$

the sign of equality holding at the end points but not elsewhere; then $F_{34}(x) \equiv F_{12}(x)$, so that (35) can be written as

$$
f(x)>F_{12}(x), \quad\left(x_{1}<x<x_{2}\right) .
$$

Fix $x_{6}$, with $a<x_{6}<x_{1}$, and let $F_{k}(x)$ satisfy

$$
F_{k}\left(x_{6}\right)=F_{12}\left(x_{6}\right), \quad F_{k}\left(x_{2}\right)=F_{12}\left(x_{2}\right)+k=f\left(x_{2}\right)+k ;
$$

in particular,

$$
F_{0}(x) \equiv F_{12}(x)
$$

Let $k$ increase continuously from $k=0$; then, for any $x>x_{6}$ in $(a, b)$, by Theorems 1 and $2, F_{k}(x)$ increases continuously. Further, it is easy to show, by Theorem 1 and the Heine-Borel Theorem, that for $k$ sufficiently large,

$$
f(x)<F_{k}(x), \quad\left(x_{1} \leqq x \leqq x_{2}\right) .
$$

By (36), (37), and (38), there is some largest $k_{0}>0$ for which

$$
f(x)=F_{k_{0}}(x)
$$

has a solution in $x_{1} \leqq x \leqq x_{2}$. Let $x_{7}$ be the largest value for which

$$
f\left(x_{7}\right)=F_{k_{0}}\left(x_{7}\right), \quad\left(x_{1} \leqq x_{7} \leqq x_{2}\right) .
$$

Since $k_{0}>0$, we have $x_{1}<x_{7}<x_{2}$.

Suppose (33) could be satisfied at $x_{7}$ for the above function $f(x)$, which is not a sub- $F(x)$ function, and for some arbitrarily small positive $h$; we shall obtain a contradiction. Let $h$, with $x_{1}<x_{8}=x_{7}-h<x_{7+h}=x_{9}<x_{2}$, be a value for which (33) is satisfied:

$$
f\left(x_{7}\right) \leqq F_{89}\left(x_{7}\right)
$$

By the choice of $k_{0}$, 


$$
\begin{aligned}
& F_{89}\left(x_{8}\right)=f\left(x_{8}\right) \leqq F_{k_{0}}\left(x_{8}\right), \\
& F_{89}\left(x_{9}\right)=f\left(x_{9}\right)<F_{k_{0}}\left(x_{9}\right),
\end{aligned}
$$

so that, by Theorem 1 and its corollary,

$$
F_{89}(x)<F_{k_{0}}(x), \quad\left(x_{8}<x<b\right) ;
$$

in particular,

$$
F_{89}\left(x_{7}\right)<F_{k_{0}}\left(x_{7}\right) .
$$

Now (41) contradicts (39) and (40).

The Rice Institute

\section{SUFFICIENT CONDITIONS FOR A NON-REGULAR PROBLEM IN THE CALCULUS OF VARIATIONS*}

\section{G. M. EWING}

1. Introduction. Given $J=\int_{x_{1}}^{x_{2}} f\left(x, y, y^{\prime}\right) d x$, it is well known that a minimizing curve satisfies the necessary conditions of Euler, Weierstrass, and Legendre, which we shall designate as I, II, and III, $\dagger$ respectively. If further, $f_{y^{\prime} y^{\prime}}\left(x, y, y^{\prime}\right) \neq 0$ on the minimizing curve, the Jacobi condition IV is necessary, while the stronger set of conditions $\mathrm{I}, \mathrm{II}_{b}^{\prime}, \mathrm{III}^{\prime}$, and $\mathrm{IV}^{\prime} \ddagger$ are sufficient for a strong relative minimum.

The purpose of this study is to obtain a set of sufficient conditions for a curve without corners along which $f_{y^{\prime} y^{\prime}}$ may have zeros. Since the classical theory gives only the necessary conditions I, II, and III, we wish to obtain a Jacobi condition; and with this in view, introduce the integral

$$
\begin{array}{r}
L \equiv \int_{x_{1}}^{x_{2}} \phi\left(x, y, y^{\prime}\right) d x, \phi\left(x, y, y^{\prime}\right) \equiv f\left(x, y, y^{\prime}\right)+k^{2}\left[y^{\prime}-e^{\prime}(x)\right]^{2}, \\
\left(x_{1} \leqq x \leqq x_{2}, k \leqq 0\right)
\end{array}
$$

by means of which we find a necessary condition that we shall call $I V_{L}^{\prime}$. Suitably strengthened, this becomes $I_{L b}^{\prime}$ and the set of conditions $\mathrm{I}, \mathrm{II}_{b}, \mathrm{III}_{b}$, and $\mathrm{IV}_{L b}^{\prime}$ are found sufficient for an improper strong relative minimum.

* Presented to the Society, November 27, 1936.

$\dagger$ G. A. Bliss, Calculus of Variations, 1925, pp. 130-132.

$\ddagger$ Bliss, loc. cit., pp. 134-135. 